\title{
Pulmonary extra-medullary hematopoiesis and pulmonary hypertension from underlying polycythemia vera: a case series
}

\author{
Inderjit Singh', Geoffrey Mikita ${ }^{2}$, Daniel Green ${ }^{3}$, Cristobal Risquez $^{4}$ and Abraham Sanders ${ }^{4}$ \\ 'Division of Pulmonary/Critical Care Medicine, Brigham and Women's Hospital, Boston, MA, USA; '2Department of Pathology, New York Presbyterian Hospital/ \\ Weill Cornell Medical College, New York, NY, USA; ${ }^{3}$ Department of Radiology, New York Presbyterian Hospital/Weill Cornell Medical College, New York, NY, \\ USA; ${ }^{4}$ Department of Medicine, Division of Pulmonary/Critical Care Medicine, New York Presbyterian Hospital/Weill Cornell Medical College, New York, NY, USA
}

\begin{abstract}
Myeloproliferative neoplasia (MPN)-associated pulmonary hypertension ( $\mathrm{PH})$ is included in group five of the most recent clinical classification of $\mathrm{PH} .{ }^{\prime}$ The MPNs are a heterogeneous group of disorders that includes disorders with primary expression of a myeloid phenotype and disorders characterized by expression of the Janus Kinase 2 (JAK2) mutation, p.V6I 7F. The latter includes essential thrombocytosis, polycythemia vera, and idiopathic myelofibrosis. ${ }^{2}$ Pulmonary extra-medullary hematopoiesis (EMH) refers to the presence of hematopoietic precursor cells in the lung. It is a rare complication associated with myelofibrosis. Here we present a case series highlighting the clinical-pathological-radiological features of pulmonary EMH and PH from underlying polycythemia vera.
\end{abstract}

\section{Keywords}

pulmonary arterial hypertension, pulmonary extra-medullary hematopoiesis, myelofibrosis

Date received: II May 2016; accepted: I3 December 2016

Pulmonary Circulation 2017; 7(I) 26I-267

DOI: 10.1 I77/20458932I7702064

\section{Case presentation}

Case I

A 69-year-old woman with a ten-year history of $J A K 2$ p.V617F-positive polycythemia vera presented with a twoyear history of progressive exertional dyspnea. On physical exam, her vital signs were normal. She had holosystolic murmur over the tricuspid area, scattered ecchymosis over limbs, and tender splenomegaly.

Her polycythemia vera was treated with hydroxyurea (500 mg daily) and later with Peg-interferon alpha $(90 \mathrm{mcg}$ weekly for eight months). However, she continued to experience worsening thrombocytopenia and tender splenomegaly. A bone marrow biopsy showed changes of postpolycythemic myelofibrosis. She was started on ruxolitinib (15 mg twice daily) along with prednisone (dose range of 15-25 mg daily). Her tender splenomegaly and functional status initially improved. However, six months later, she experienced worsening exertional dyspnea. A ventilation- perfusion (VQ) scan showed very low probability for pulmonary embolism and chest CT showed diffuse bilateral ground glass opacities (GGOs) (Fig. 1a). A $\mathrm{T}_{\mathrm{C}} \mathrm{M}$-labeled colloid scan showed no evidence of EMH. Surgical lung biopsy of the left lower lobe showed an interstitial pneumonitis-like EMH (Fig. 1b-d). Single fraction whole-lung radiation was initiated (1000 cGY). Her ruxolitinib was held for six weeks because of worsening anemia and thrombocytopenia from radiation therapy. During this period, repeat echocardiogram was unchanged from four months prior. Her ruxolitinib was restarted following improvement in her cell counts. However, she continued to experience worsening exertional dyspnea. Repeat chest CT demonstrated progressive bilateral GGOs. A bronchoscopy with broncho-alveolar lavage (BAL) of the right middle lobe ruled out infection. A repeat echocardiogram study

Corresponding author:

Cristobal Risquez, 425 E 6Ist St, New York, NY 10065, USA.

Email: cfr9002@nyp.org 

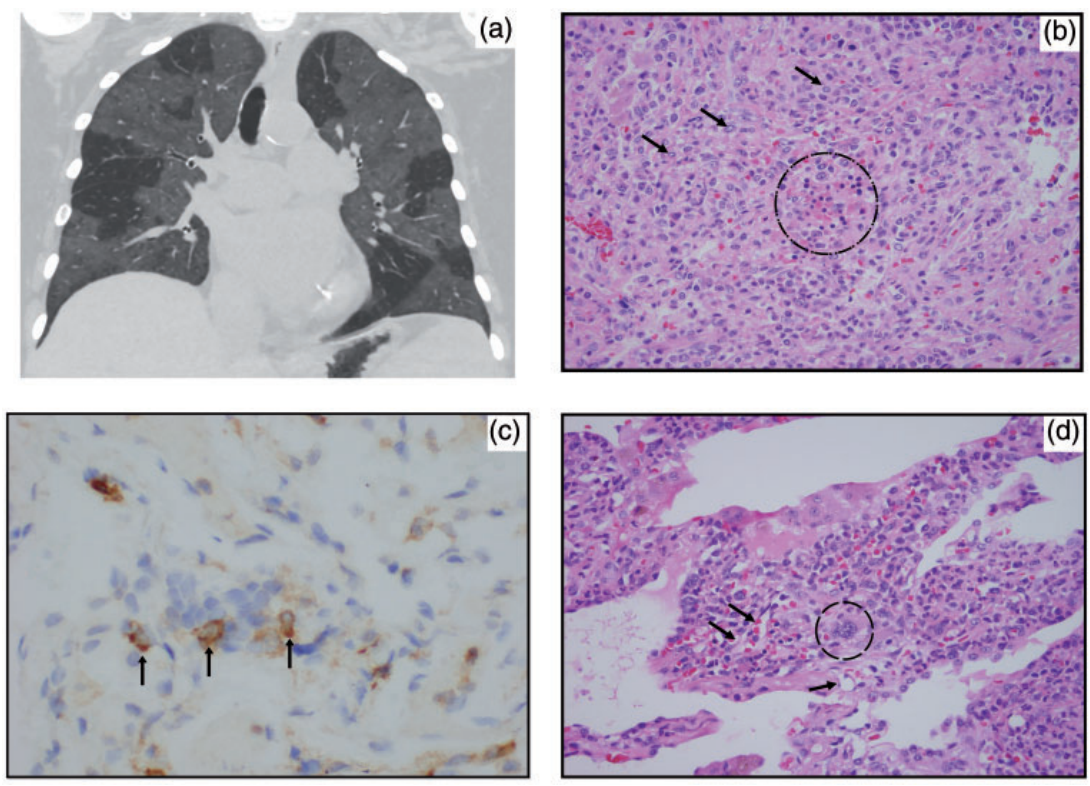

Fig. I (a) Coronal CT image shows bilateral GGOs. Sharp demarcation between affected lung and normal lung suggests a lobular distribution. (b) Erythroid precursors (dotted circle) vaguely forming a colony. Myeloid precursors are also present (arrows point out representatives).

(c) Myeloid specific marker CD33 stained cells occupying the interstitium. Vasculocentricity is present. (d) A megakaryocyte adjacent to small vessels (dotted circle) around megakaryocyte; (arrows) pointing out small neighboring vessels.

showed severely dilated right ventricle with severe PH. A subsequent right heart catheterization (RHC) demonstrated moderate pre-capillary $\mathrm{PH}$ (Table 2). She was started on sildenafil ( $20 \mathrm{mg}$ three times daily). However, despite initial improvement in her dyspnea, she died several months later.

\section{Case 2}

A 72-year-old man with $J A K 2$ p.V617F-positive secondary myelofibrosis was referred for bronchoscopy following an abnormal chest radiograph as part of pre-allogeneic stem cell transplant workup. Review of systems was notable for increasing fatigability and dyspnea on exertion over the past few months.

He was diagnosed with JAK2-positive polycythemia vera 25 years ago. He was treated with hydroxyurea $(500 \mathrm{mg}$ daily), Peg-interferon alpha (135 mcg weekly), and imatinib (300 mg daily). Despite this, he continued to have worsening anemia, thrombocytopenia, and increasing tender splenomegaly. He was ultimately switched to ruxolitinib $(20 \mathrm{mg}$ in the morning and $15 \mathrm{mg}$ at night) and 6-mercaptopurine (50 mg three times a week). However, he remained transfusion dependent and the decision was made to proceed with allogeneic stem cell transplantation.

On presentation, his vital signs were normal. On exam, he had scattered ecchymosis over his upper limbs and trunk, and tender splenomegaly. Initial investigations were notable for leukocytosis of $40.7 \times 10^{3} / \mathrm{uL}$ (with $39 \%$ neutrophils, $7 \%$ lymphocytes, $13 \%$ myelocytes, $5 \%$ basophils), hemoglobin of $7.8 \mathrm{~g} / \mathrm{dL}$, and platelet count of $40 \times 10^{3} / \mathrm{uL}$. Chest X-ray demonstrated right greater than left peri-hilar opacity and chest CT showed bilateral patchy foci of GG attenuation with associated interlobular septal thickening (Fig. 2a). Subsequent bronchoscopy demonstrated bloody secretions in bilateral airways with no active bleeding. BAL aliquots of right upper and medial lobes returned progressively bloodier consistent with diffuse alveolar hemorrhage. The microbiologic analysis of the BAL was negative for infection. A surgical lung biopsy of the right upper lobe was then performed. This showed multi-focal EMH and pulmonary arteriopathy with medial thickening (Fig. 2b and c). The patient also had an echocardiogram performed that demonstrated moderate $\mathrm{PH}$ with normal right ventricular function and size. A subsequent RHC demonstrated mild pre-capillary PH (Table 2).

The patient is currently awaiting pre-allogeneic stem cell transplantation induction therapy with decitabine.

\section{Case 3}

A 63-year-old woman with a history of JAK2 p.V617Fpositive secondary myelofibrosis presented with a twoweek history of fevers, chills, and productive cough of yellow sputum. Review of systems was notable for a twoyear history of increasing lethargy, worsening exertional dyspnea, and non-productive cough. Her past medical history was notable for polycythemia vera diagnosed at age 36 years which evolved into secondary myelofibrosis three years ago. She has been on several different therapies 

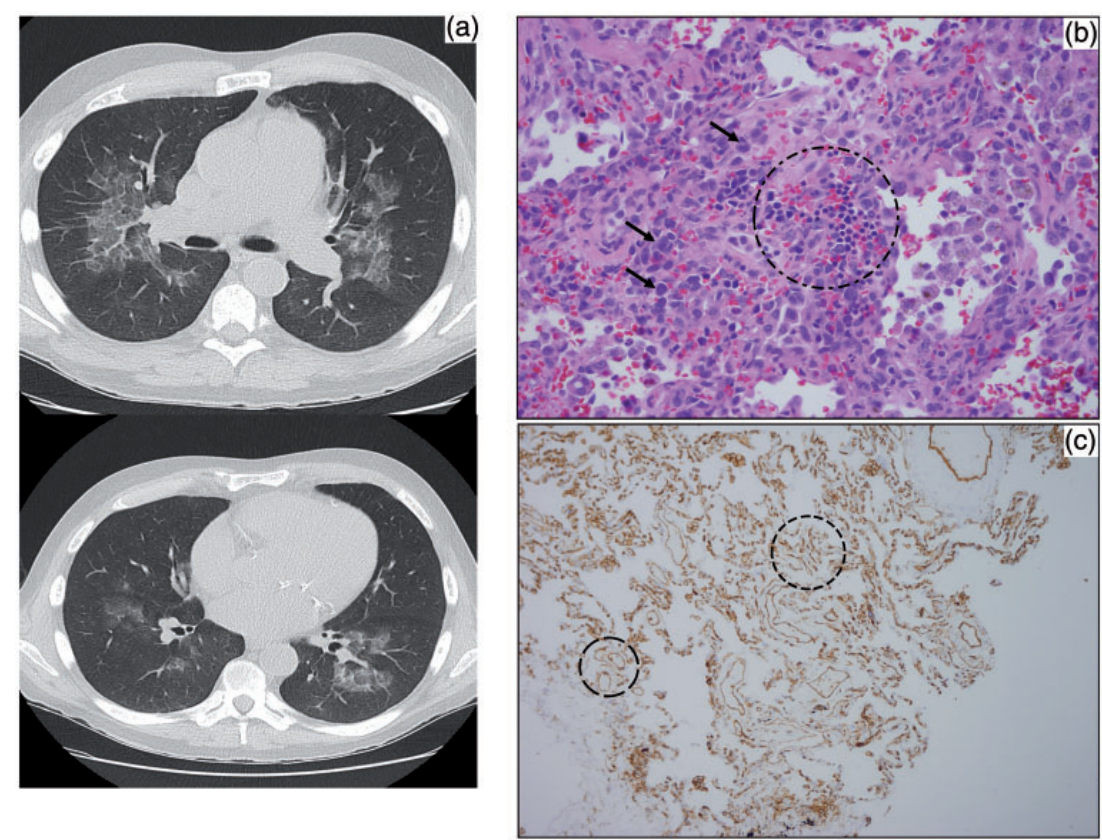

Fig. 2 (a) Axial CT image shows bilateral GGOs with underlying interlobular septal thickening - the "crazy paving” sign. (b) Erythroid colony (circle) with myeloid precursors (arrows point out representatives) in the wall of airway. (c) CD3I immunostain showing increased small vessel density (circles).

including hydroxyurea, danazol, thalidomide, and elotuzumab. Over the past one year she had been on a clinical trial drug, CYT 387.

On admission, her temperature was $39.2^{\circ} \mathrm{C}$, heart rate $101 \mathrm{bpm}$, blood pressure $121 / 67 \mathrm{mmHg}$, oxygen saturation $97 \%$ breathing ambient air, and respiratory rate of 16 breaths/min. Physical exam was notable for scattered ecchymosis over her trunk and limbs, and tender splenomegaly. Initial investigation was notable for increasing leukocytosis of $19.1 \times 10^{3} / \mathrm{uL}\left(3\right.$ months prior: $9.7 \times 10^{3} / \mathrm{uL}$ ) with $35 \%$ neutrophils, hemoglobin of $10.7 \mathrm{~g} / \mathrm{dL}$, and platelet count of $66 \times 10^{3} / \mathrm{uL}$ (3 months prior $\left.28 \times 10^{3} / \mathrm{uL}\right)$. Admission chest X-ray demonstrated interval development of patchy air-space opacity in the lingula. She was treated for suspected pneumonia with ceftriaxone and azithromycin. She also had a bronchoscopy with BAL of the lingula. The BAL study was negative for infection. She was discharged and had a brief period of clinical improvement following completion of her antibiotic course. However, she re-presented several weeks later with two episodes of scant hemoptysis. A repeat chest CT showed diffuse nodular interstitial thickening with a few small consolidative areas (Fig. 3a). Repeat bronchoscopy did not demonstrate bleeding or infection. A surgical lung biopsy of the right lower lobe showed intra-pulmonary histiocytoid Sweet's syndrome with a component of EMH (Fig. $3 \mathrm{~b}$ and c). At this juncture, she was commenced on prednisone.

Approximately six months later, her secondary myelofibrosis transformed into acute myeloid leukemia. Her investigational drug was discontinued and she was treated initially with hydroxyurea and later with decitabine.
However, her cough and exertional dyspnea continued to worsen and repeat chest CT demonstrated new patchy consolidations of the right upper and left lower lobes. An echocardiogram performed showed mild $\mathrm{PH}$ with normal right and left ventricular size and function (Table 2). She continued to decline clinically and died soon after.

\section{Discussion}

Most cases of EMH are tied to underlying chronic MPN or hemoglobinopathies such as thalassemia and sickle cell anemia. EMH commonly involves the liver and spleen but may occur in the lungs, pleura, lymph nodes, urogenital system, and in the para-spinal or epidural spaces. ${ }^{3}$ Intrapulmonary involvement is rare, with approximately 40 cases described. ${ }^{4}$ The pathogenesis of pulmonary EMH is poorly understood. It may represent differentiation of embryonic hematopoietic stem cells or the abnormal release of marrow precursors into the circulation. The latter is supported by the $400 \times$ increase in number of circulating CD34+ hematopoietic precursor cells in patients with primary myelofibrosis. This forces the expansion of hematopoietic tissue in areas other than the liver and spleen. ${ }^{5}$

Chest CT is the main imaging modality in the evaluation of pulmonary EMH, though findings are non-specific. Prior case reports demonstrate varied imaging appearances, which include pulmonary nodules and masses, consolidations, septal line thickening, and pulmonary fibrosis. ${ }^{6}$ Nodules can be solitary or multiple, bilateral or unilateral, and calcified or non-calcified. ${ }^{3}$ In the three cases presented above, the predominant findings are interlobular septal thickening 

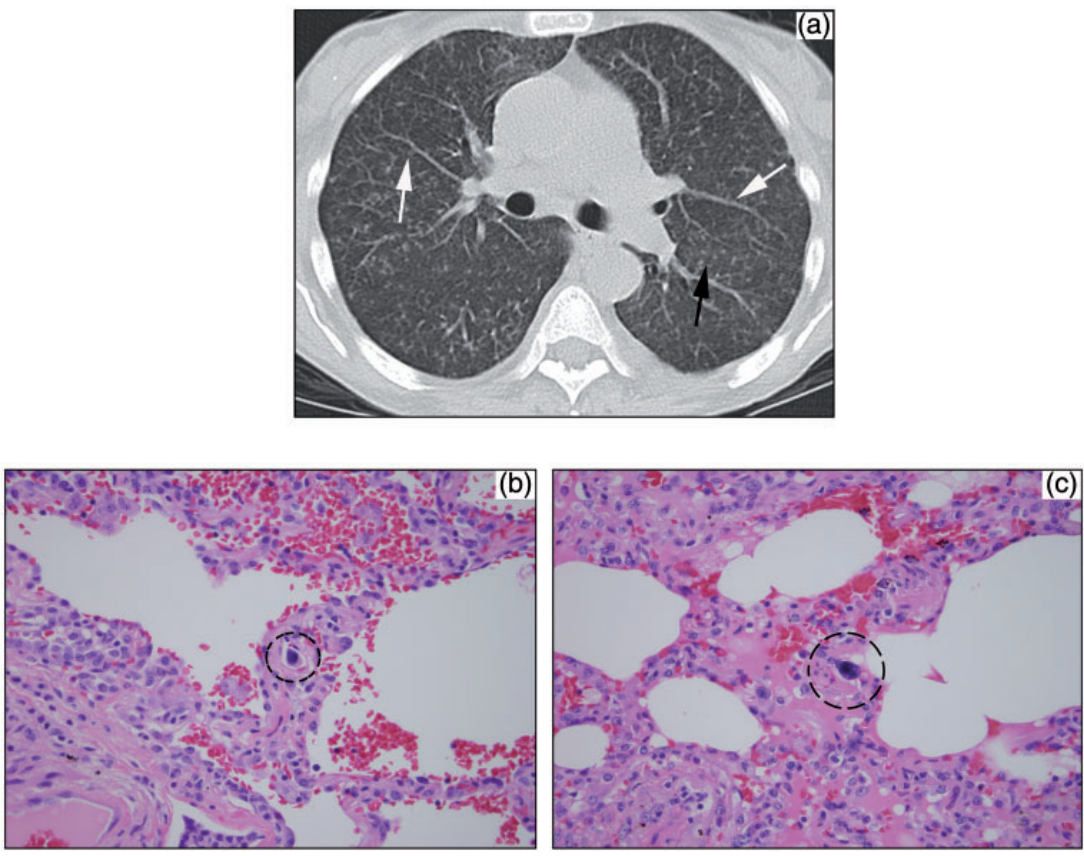

Fig. 3 (a) Axial CT image shows diffuse interlobular septal thickening and tiny I-2 mm nodules studding the left major fissure (black arrow) and peribronchovascular interstitium (white arrows). (b, c) Megakaryocyte (circle) within interstitial lung capillary in two different lung sections.

and GGOs. These two findings are superimposed in one case, a combination commonly referred to as the "crazy paving" sign. While one case demonstrates innumerable 1$2 \mathrm{~mm}$ interstitial nodules, none depict larger nodules or masses, and there is no example of pulmonary fibrosis. To our knowledge, these are the first cases of pulmonary EMH with GGOs, which probably correspond to the histologic finding of intra-lobular septal thickening. These microscopic septa are below the resolution of CT and imperceptible when normal. When thickened, the aggregate appearance is GG attenuation rather than multiple discrete lines, unlike thickened interlobular septa, which are larger and clearly linear. In the setting of thrombocytopenia, alveolar hemorrhage may contribute to GGOs. In areas of more focal or profuse hemorrhage, small consolidations may be present. In case 2 depicting the "crazy paving" sign, the main diagnostic consideration prior to biopsy was secondary pulmonary alveolar proteinosis, which is also associated with myelodysplastic syndrome. ${ }^{7}$ The most common site of EMH in the thorax is the posterior mediastinum. ${ }^{8}$ Solitary or multilevel para-spinal masses, with or without fat, are seen in several causes of chronic anemia. The presence of para-spinal masses would lend support to a diagnosis of pulmonary EMH with the appropriate findings; however, there was no posterior mediastinal involvement in our three cases. Pleural EMH is considered particularly rare and also was not present. ${ }^{8}$ In addition to standard chest $\mathrm{CT}$, radionuclide imaging can be used to detect sites of EMH using radiotracers that normally distribute in bone marrow. Technetium-99 $\mathrm{m}$ sulfur colloid and technetium$99 \mathrm{~m}$ anti-CD66 monoclonal antibody have both demonstrated pulmonary uptake in the presence of EMH. ${ }^{9}$
Table I. Proposed mechanism of pulmonary hypertension in myeloproliferative neoplasms.

Thrombocytosis with increased platelet activation and secretion of PDGF or serotonin

Chronic thrombo-embolic pulmonary hypertension (CTEPH)

Thrombotic occlusion of pulmonary vasculature

Splenectomy

Portal hypertension

High output states

Drug induced

Pulmonary veno-occlusive disease (anagrelide)

Pulmonary arterial hypertension (dasatinib and interferon alpha/ beta)

Aberrant JAK/Src-STAT3 signaling pathway

Promotes inflammation

Promotes abnormal hyper-proliferative state

Enhances abnormal angiogenesis

Depletes endothelial nitric oxide stores

\section{Pulmonary vascular remodeling by myeloid cells}

PDGF, platelet-derived growth factor; JAK, Janus Kinase; STAT, signal transducer and activator of transcription.

False negatives occur if there is little medullary stroma present or if there is low cell activity. ${ }^{5}$

There are several pathophysiologic mechanisms implicated in the development of $\mathrm{PH}$ in patients with MPN (Table 1). ${ }^{10}$ Drugs that have been implicated with $\mathrm{PH}$ include anagrelide, ${ }^{10}$ ruxolitinib,${ }^{11}$ dasatinib, ${ }^{1}$ and interferon alpha. ${ }^{12}$ Dasatinib and interferon therapy are regarded as likely and possible causes of $\mathrm{PH}$, respectively. ${ }^{1}$ Ruxolitinib, a selective JAK 1 and 2 inhibitor, improves splenomegaly, disease-related symptoms, and quality of life in patients with 
Table 2. Patient demographic, chest CT features, pathological findings, echocardiographic, and RHC data.

\begin{tabular}{|c|c|c|c|}
\hline & Case I & Case 2 & Case 3 \\
\hline Age (years) & 60 & 72 & 63 \\
\hline Sex & Female & Male & Female \\
\hline \multicolumn{4}{|l|}{$C T$ of chest } \\
\hline Findings and distribution & $\begin{array}{l}\text { Diffuse, bilateral, } \\
\text { lobular GGOs }\end{array}$ & $\begin{array}{l}\text { Diffuse, bilateral, lobular } \\
\text { GGOs and interlobular } \\
\text { septal thickening }\end{array}$ & $\begin{array}{l}\text { Diffuse, bilateral, } \\
\text { interstitial interlobular septal } \\
\text { thickening with } \\
\text { peri-lymphatic nodules }\end{array}$ \\
\hline Fibrosis, pleural, or para-spinal involvement & No & No & No \\
\hline \multicolumn{4}{|l|}{ Pathological findings } \\
\hline EMH & Yes & Yes & Yes \\
\hline Pulmonary arteriopathy & No & Yes & No \\
\hline Other features & - & - & $\begin{array}{l}\text { Intra-pulmonary histiocytoid } \\
\text { Sweet's syndrome }\end{array}$ \\
\hline \multicolumn{4}{|l|}{ Echocardiogram } \\
\hline TR max velocity $(\mathrm{m} / \mathrm{s})$ & 4 & - & 3.2 \\
\hline Estimated PAP (mmHg) & 76.2 & 54 & 44.3 \\
\hline TAPSE $(\mathrm{cm})$ & 2.4 & 2.7 & 2.1 \\
\hline RV size & Severely dilated & Normal & Normal \\
\hline RV function & Normal & Normal & Normal \\
\hline \multicolumn{4}{|l|}{ Right heart catheterization (baseline hemodynamics) } \\
\hline $\mathrm{RAP}(\mathrm{mmHg})$ & 0 & 6 & \multirow{5}{*}{$\begin{array}{l}\text { Right heart catheterization } \\
\text { not performed }\end{array}$} \\
\hline mPAP $(\mathrm{mmHg})$ & 32 & 28 & \\
\hline PCWP (mmHg) & 12 & 10 & \\
\hline PVR (WU) & 4.4 & 3.63 & \\
\hline Cardiac output (L/min) & 4.5 & 4.9 & \\
\hline
\end{tabular}

mPAP, mean pulmonary arterial pressure; PAP, pulmonary arterial pressure; PCWP, pulmonary capillary wedge pressure; PVR, pulmonary vascular resistance; RAP, right atrial pressure; TAPSE, tricuspid annular plane systolic excursion; TR, tricuspid regurgitation; WU, Woods unit.

myelofibrosis. ${ }^{13}$ There has been a single case report implicating ruxolitinib with worsening $\mathrm{PH}$ in a patient with $J A K 2$ p.V617F-positive idiopathic myelofibrosis. The patient developed worsening exertional dyspnea two and six weeks following ruxolitinib commencement with subsequent symptomatic and echocardiographic improvement on withdrawal of the drug on two separate occasions. ${ }^{11}$ Our patient in case 1 developed worsening exertional dyspnea six months after starting ruxolitinib. The development of her $\mathrm{PH}$ coincided with worsening leukocytosis and radiographic progression of disease. Furthermore, there was lack of echocardiographic improvement following ruxolitinib cessation. The lack of echocardiography (TTE) prior to initiation of ruxolitinib is a limitation in case 2. However, the onset of his symptoms was about two years following ruxolitinib and coincided with radiographic evidence of disease. Peg-interferon alpha therapy is unlikely to be the cause of $\mathrm{PH}$ in our patients. The patients in cases 1 and 2 did not have the usual co-morbidities seen with interferon therapy-associated pulmonary arterial hypertension (PAH), such as chronic hepatitis $\mathrm{C}$ or HIV co-infection. ${ }^{12}$ Furthermore, they were off their Peg-interferon alpha treatment for more than one year prior to their symptom onset and $\mathrm{PH}$ progression. The true presence of pulmonary hypertension in case 3 is uncertain owing to the absence of RHC data. The echocardiogram in case 3 showed mild $\mathrm{PH}$ with a normal right ventricular size and function. Studies that use TTE as the primary diagnostic tool are hampered by inaccuracies leading to frequent over- or under-estimation of true right ventricular systolic pressure ${ }^{14-17}$ and are also discordant from the gold standard, RHC findings. ${ }^{18}$ Table 2 summarizes the chest $\mathrm{CT}$ features, pathological findings, echocardiographic, and RHC data of our three patients.

On a molecular level, aberrancy in the Janus Kinase (JAK)/Src-Signal Transducer and Activator of Transcription 3 (JAK/Src-STAT3) signaling pathway may also contribute to the pathogenesis of $\mathrm{PH}$ in myelofibrosis (Table 2). All three patients with polycythemia vera had JAK2 p.V617F mutation and later developed myelofibrosis. JAK-2 expression has been demonstrated in limited cutaneous sclerosis-associated PAH but not in idiopathic PAH. ${ }^{19}$ Increase in Src and Src-related proteins are found in PAHpulmonary arterial smooth muscle cells (PASMC) and in hypoxia and monocrotaline experimental models of $\mathrm{PAH}{ }^{20}$ Src and JAK proteins phosphorylate and activate STAT3 protein in response to various cytokines. ${ }^{21}$ These cytokines, which include endothelin-1, angiotensin $\mathrm{II}^{22}$ and platelet-derived growth factor (PDGF) ${ }^{23}$ are 
commonly implicated in the pathogenesis of PAH. The increased and sustained STAT3 phosphorylation potentially contributes to pulmonary hypertension by promoting abnormal vascular endothelial cell hyper-proliferation, ${ }^{24}$ by promoting inflammation ${ }^{21}$ and angiogenesis, ${ }^{21}$ and by depleting pulmonary vascular endothelial cells (PAECs) nitric oxide stores. ${ }^{25}$

In idiopathic PAH patients, increased numbers of activated STAT3 are seen in plexiform lesions, intimal lesions, and in the endothelium of small pulmonary arteries. Inhibition of STAT3 phosphorylation was shown to reduce the proliferation of PAECs in these lesions. ${ }^{24}$ Meanwhile, inhibition of JAK-1 and JAK-2 by ruxolitinib increases NO levels and improves serum biomarkers of $\mathrm{PH}$ in patients with myelofibrosis and echocardiographic evidence of $\mathrm{PH}$. This increase in NO is related to the increase in NO-inducing cytokine (IFN-gamma) and reduction in NO-inhibiting cytokines (TNF, IL-4, and IL-10). ${ }^{25}$ However, the use of other tyrosine kinase inhibitors (TKI) in PH has yielded mixed results. Dasatanib has been implicated as a likely cause of drug-induced $\mathrm{PH} .{ }^{1}$ Imatinib was shown to improve pulmonary hemodynamics and exercise capacity in patients with idiopathic $\mathrm{PAH} .{ }^{26}$ The exact mechanism of dasatinib-induced $\mathrm{PH}$ is not well understood. Among patients with dasatinib-induced $\mathrm{PH}$, those given nilotinib following dasatinib discontinuation did not develop disease recurrence. This suggests that dasatinibinduced $\mathrm{PH}$ is perhaps a specific complication of dasatinib use rather than a TKI-class related effect. ${ }^{27}$ There is also increasing evidence supporting the role of myeloid cells in the pathogenesis of PAH. ${ }^{28-31}$ The three patients had myeloid metaplasia, and myeloid hematopoietic precursors occupied the pulmonary interstitium and showed vasculocentricity. In patients with idiopathic $\mathrm{PAH}$, there is an increase in myeloid progenitor cell accumulation in the remodeled pulmonary arteries. ${ }^{29}$ Recently, Yan et al. demonstrated that control mice transplanted with bone marrow that expressed the $B M P R 2^{R 899 X}$ mutation developed neo-muscularization of the pulmonary vasculature, while engraftment of $B M P R 2^{R 899 X}$ transgenic mice with control bone marrow attenuated $\mathrm{PH}$ development. ${ }^{31}$ These studies suggest that myeloid cells play a key role in the pathogenesis of $\mathrm{PH}$.

\section{Conclusion}

Pulmonary EMH in myelofibrosis with resulting $\mathrm{PH}$ is a rare clinical entity. Our series expands on prior reports and demonstrates consistent clinical-pathologic-radiological correlates useful to clinicians and pathologists in their assessment of patients with MPN who present with pulmonary symptoms suggestive of EMH. The treatment of chronic MPN-associated PH should be directed towards the underlying cause. When PH is diagnosed, associated conditions such hypoxia, anemia, and heart failure should be treated in conjunction with appropriate cyto-reductive agents. PH-specific pharmacotherapy should be reserved for patients who continue to demonstrate exhausted cardio-pulmonary reserve despite conventional therapies. Unfortunately, the prognosis of patients with chronic MPN-associated PH remains poor with a median survival time of 18 months. $^{32}$

\section{Conflict of interest}

The author(s) declare that there is no conflict of interest.

\section{Funding}

This research received no specific grant from any funding agency in the public, commercial, or not-for-profit sectors.

\section{References}

1. Simonneau G, Gatzoulis MA, Adatia I, et al. Updated clinical classification of pulmonary hypertension. $J$ Am Coll Cardiol 2013; 62(Suppl. 25): D34-41.

2. Adir Y, Elia D and Harari S. Pulmonary hypertension in patients with chronic myeloproliferative disorders. Eur Respir Rev 2015; 24(137): 400-410.

3. Bowling MR, Cauthen CG, Perry CD, et al. Pulmonary extramedullary hematopoiesis. $J$ Thorac Imaging 2008; 23(2): $138-141$.

4. Trow TK, Argento AC, Rubinowitz AN, et al. A 71-year-old woman with myelofibrosis, hypoxemia, and pulmonary hypertension. Chest 2010; 138(6): 1506-1510.

5. Barosi G, Viarengo G, Pecci A, et al. Diagnostic and clinical relevance of the number of circulating $\mathrm{CD} 34(+)$ cells in myelofibrosis with myeloid metaplasia. Blood 2001; 98(12): 3249-3255.

6. Asakura S and Colby TV. Agnogenic myeloid metaplasia with extramedullary hematopoiesis and fibrosis in the lung. Report of two cases. Chest 1994; 105(6): 1866-1868.

7. Ohnishi T, Yamada G, Shijubo N, et al. Secondary pulmonary alveolar proteinosis associated with myelodysplastic syndrome. Intern Med 2003; 42(2): 187-190.

8. Koch CA, Li CY, Mesa RA, et al. Nonhepatosplenic extramedullary hematopoiesis: associated diseases, pathology, clinical course, and treatment. Mayo Clin Proc 2003; 78(10): $1223-1233$.

9. Rumi E, Passamonti F, Boveri E, et al. Dyspnea secondary to pulmonary hematopoiesis as presenting symptom of myelofibrosis with myeloid metaplasia. Am J Hematol 2006; 81(2): 124-127.

10. Lahm $\mathrm{T}$ and Chakinala MM. World Health Organization group 5 pulmonary hypertension. Clin Chest Med 2013; 34(4): 753-778.

11. Low AT, Howard L, Harrison C, et al. Pulmonary arterial hypertension exacerbated by ruxolitinib. Haematologica 2015; 100(6): e244-245.

12. Savale L, Sattler C, Günther S, et al. Pulmonary arterial hypertension in patients treated with interferon. Eur Respir J 2014; 44(6): 1627-1634.

13. Harrison C, Kiladjian JJ, Al-Ali HK, et al. JAK inhibition with ruxolitinib versus best available therapy for myelofibrosis. $N$ Engl J Med 2012; 366(9): 787-798.

14. Arcasoy SM, Christie JD, Ferrari VA, et al. Echocardiographic assessment of pulmonary hypertension in 
patients with advanced lung disease. Am J Respir Crit Care Med 2003; 167(5): 735-740.

15. Fisher MR, Criner GJ, Fishman AP, et al. Estimating pulmonary artery pressures by echocardiography in patients with emphysema. Eur Respir J 2007; 30(5): 914-921.

16. Hsu VM, Moreyra AE, Wilson AC, et al. Assessment of pulmonary arterial hypertension in patients with systemic sclerosis: comparison of noninvasive tests with results of right-heart catheterization. $J$ Rheumatol 2008; 35(3): 458-465.

17. Bourbonnais JM and Samavati L. Clinical predictors of pulmonary hypertension in sarcoidosis. Eur Respir $J$ 2008; 32(2): 296-302.

18. Nathan SD, Shlobin OA, Barnett SD, et al. Right ventricular systolic pressure by echocardiography as a predictor of pulmonary hypertension in idiopathic pulmonary fibrosis. Respir Med 2008; 102(9): 1305-1310.

19. Christmann RB, Hayes E, Pendergrass S, et al. Interferon and alternative activation of monocyte/macrophages in systemic sclerosis-associated pulmonary arterial hypertension. Arthritis Rheum 2011; 63(6): 1718-1728.

20. Courboulin A, Paulin R, Giguère NJ, et al. Role for miR-204 in human pulmonary arterial hypertension. J Exp Med 2011; 208(3): 535-548.

21. Paulin R, Meloche $\mathrm{J}$ and Bonnet S. STAT3 signaling in pulmonary arterial hypertension. JAKSTAT 2012; 1(4): 223-233.

22. Banes-Berceli AK, Ketsawatsomkron P, Ogbi S, et al. Angiotensin II and endothelin-1 augment the vascular complications of diabetes via JAK2 activation. Am J Physiol Heart Circ Physiol 2007; 293(2): H1291-1299.

23. Darnell JE. STATs and gene regulation. Science 1997; 277(5332): 1630-1635.
24. Masri FA, Xu W, Comhair SA, et al. Hyperproliferative apoptosis-resistant endothelial cells in idiopathic pulmonary arterial hypertension. Am J Physiol Lung Cell Mol Physiol 2007; 293(3): L548-554.

25. Tabarroki A, Lindner DJ, Visconte V, et al. Ruxolitinib leads to improvement of pulmonary hypertension in patients with myelofibrosis. Leukemia 2014; 28(7): 1486-1493.

26. Hoeper MM, Barst RJ, Bourge RC, et al. Imatinib mesylate as add-on therapy for pulmonary arterial hypertension: results of the randomized IMPRES study. Circulation 2013; 127(10): $1128-1138$.

27. Godinas L, Guignabert C, Seferian A, et al. Tyrosine kinase inhibitors in pulmonary arterial hypertension: a double-edge sword? Semin Respir Crit Care Med 2013; 34(5): 714-724.

28. Farha $\mathrm{S}$, Asosingh $\mathrm{K}, \mathrm{Xu} \mathrm{W}$, et al. Hypoxia-inducible factors in human pulmonary arterial hypertension: a link to the intrinsic myeloid abnormalities. Blood 2011; 117(13): 3485-3493.

29. Montani D, Perros F, Gambaryan N, et al. C-kit-positive cells accumulate in remodeled vessels of idiopathic pulmonary arterial hypertension. Am J Respir Crit Care Med 2011; 184(1): 116-123.

30. Asosingh K, Farha S, Lichtin A, et al. Pulmonary vascular disease in mice xenografted with human BM progenitors from patients with pulmonary arterial hypertension. Blood 2012; 120(6): 1218-1227.

31. Yan L, Chen X, Talati M, et al. Bone marrow-derived cells contribute to the pathogenesis of pulmonary arterial hypertension. Am J Respir Crit Care Med 2016; 193(8): 898-909.

32. Dingli D, Utz JP, Krowka MJ, et al. Unexplained pulmonary hypertension in chronic myeloproliferative disorders. Chest 2001; 120(3): 801-808. 